\title{
X-ray ptychographic topography: A robust nondestructive tool for strain imaging
}

\author{
Mariana Verezhak $\odot,{ }^{1, *}$ Steven Van Petegem $\odot,{ }^{1}$ Angel Rodriguez-Fernandez, ${ }^{2}$ Pierre Godard $\odot,{ }^{3}$ Klaus Wakonig,,${ }^{1,4}$ \\ Dmitry Karpov, ${ }^{1}$ Vincent L. R. Jacques, ${ }^{5}$ Andreas Menzel $\odot,{ }^{1}$ Ludovic Thilly, ${ }^{3}$ and Ana Diaz ${ }^{1}$ \\ ${ }^{1}$ Paul Scherrer Institute, Forschungsstrasse 111, 5232 Villigen PSI, Switzerland \\ ${ }^{2}$ European XFEL, Holzkoppel 4, 22869 Schenefeld, Germany \\ ${ }^{3}$ Institut Pprime, CNRS-University of Poitiers-ENSMA, SP2MI, Futuroscope, France \\ ${ }^{4}$ ETH and University of Zürich, Institute for Biomedical Engineering, 8093 Zürich, Switzerland \\ ${ }^{5}$ Laboratoire de Physique des Solides UMR8502, CNRS, Université Paris-Saclay, 91405, Orsay, France
}

(Received 3 July 2020; revised 16 February 2021; accepted 5 April 2021; published 30 April 2021)

\begin{abstract}
Strain and defects in crystalline materials are responsible for the distinct mechanical, electric, and magnetic properties of a desired material, making their study an essential task in material characterization, fabrication, and design. Existing techniques for the visualization of strain fields, such as transmission electron microscopy and diffraction, are destructive and limited to thin slices of the materials. On the other hand, nondestructive x-ray imaging methods either have a reduced resolution or are not robust enough for a broad range of applications. Here we present $\mathrm{x}$-ray ptychographic topography, a method for strain imaging, and demonstrate its use on an InSb micropillar after microcompression, where the strained region is visualized with a spatial resolution of $30 \mathrm{~nm}$. Thereby, x-ray ptychographic topography proves itself as a robust nondestructive approach for the imaging of strain fields within bulk crystalline specimens with a spatial resolution of a few tens of nanometers.
\end{abstract}

DOI: 10.1103/PhysRevB.103.144107

\section{INTRODUCTION}

Progress in advanced materials, from lightweight composites to biomedical technology, relies on understanding the relationship between the structure and properties of a material. In addition to the chemical composition and crystalline lattice, strain fields caused by defects also regulate material properties. Therefore, the detection and characterization of strain and its relation to the type and concentration of defects in the crystal at the nanoscale is an essential, albeit challenging task.

Transmission electron microscopy (TEM) provides imaging of crystalline defects with atomic spatial resolution. However, due to the limited penetration depth of electrons, invasive sample preparation of thin sections is required, which can modify the strain fields to be analyzed [1]. X-ray diffraction, on the other hand, is a noninvasive alternative which is sensitive to atomic displacements [2]. X-ray topography (XRT) $[3,4]$ has been routinely used for imaging the defect microstructure in the micrometer- to centimeter-sized crystals based on the diffraction contrast. In XRT, the image contrast comes from variations in the crystal lattice spacing and/or orientation, with the resolution limited by the detector pixel size.

To achieve higher spatial resolution with $\mathrm{x}$ rays, $\mathrm{x}$-ray focusing optics were introduced, as for example in Laue $\mathrm{x}$ ray microdiffraction [5]. Here, the diffraction patterns are

*Corresponding author: mariana.verezhak@psi.ch

Published by the American Physical Society under the terms of the Creative Commons Attribution 4.0 International license. Further distribution of this work must maintain attribution to the author(s) and the published article's title, journal citation, and DOI. obtained with a polychromatic x-ray beam and each diffraction spot comes from a different crystalline plane. The peak position depends on the average crystal orientation and unit cell parameters, while the width provides information on the strain gradient within the illuminated volume. Therefore, any change in peak positions and/or widths is the footprint of a specific strain field in the crystal caused by the presence of particular defects or lattice distortions. In such experiments, spatial resolution is achieved by focusing the beam and raster scanning the sample at different positions [6]. Monochromatic $\mathrm{x}$-ray diffraction microscopy in full field [7] and scanning mode [8,9] have also been able to reveal the strain field in crystalline samples with the spatial resolution limited by the focusing lens.

With advancements in synchrotron brilliance and degree of spatial coherence, probing individual crystalline defects like dislocations became possible [10]. Coherent x-ray diffraction microscopy has enabled nanometer spatial resolution beyond focusing optics limitations [11,12]. Bragg coherent diffraction imaging (BCDI) is based on measuring the far-field diffraction patterns of a fully illuminated crystal placed in the Bragg condition and subsequent application of a phase retrieval algorithm for image reconstruction. Thus, BCDI provides 3D maps of the atomic displacement field within a small, isolated nanocrystal with a spatial resolution of a few tens of nanometers [13]. On the other hand, ptychographic methods, based on measuring a series of diffraction patterns at a set of overlapping areas [14], enable the application of coherent methods to larger crystalline specimens. Bragg ptychography is also capable of imaging strain; however, the strict requirements on the specimen translation and beam stability make the technique difficult to apply, especially in 3D [15]. Bragg projection ptychography [16] has more relaxed stability 


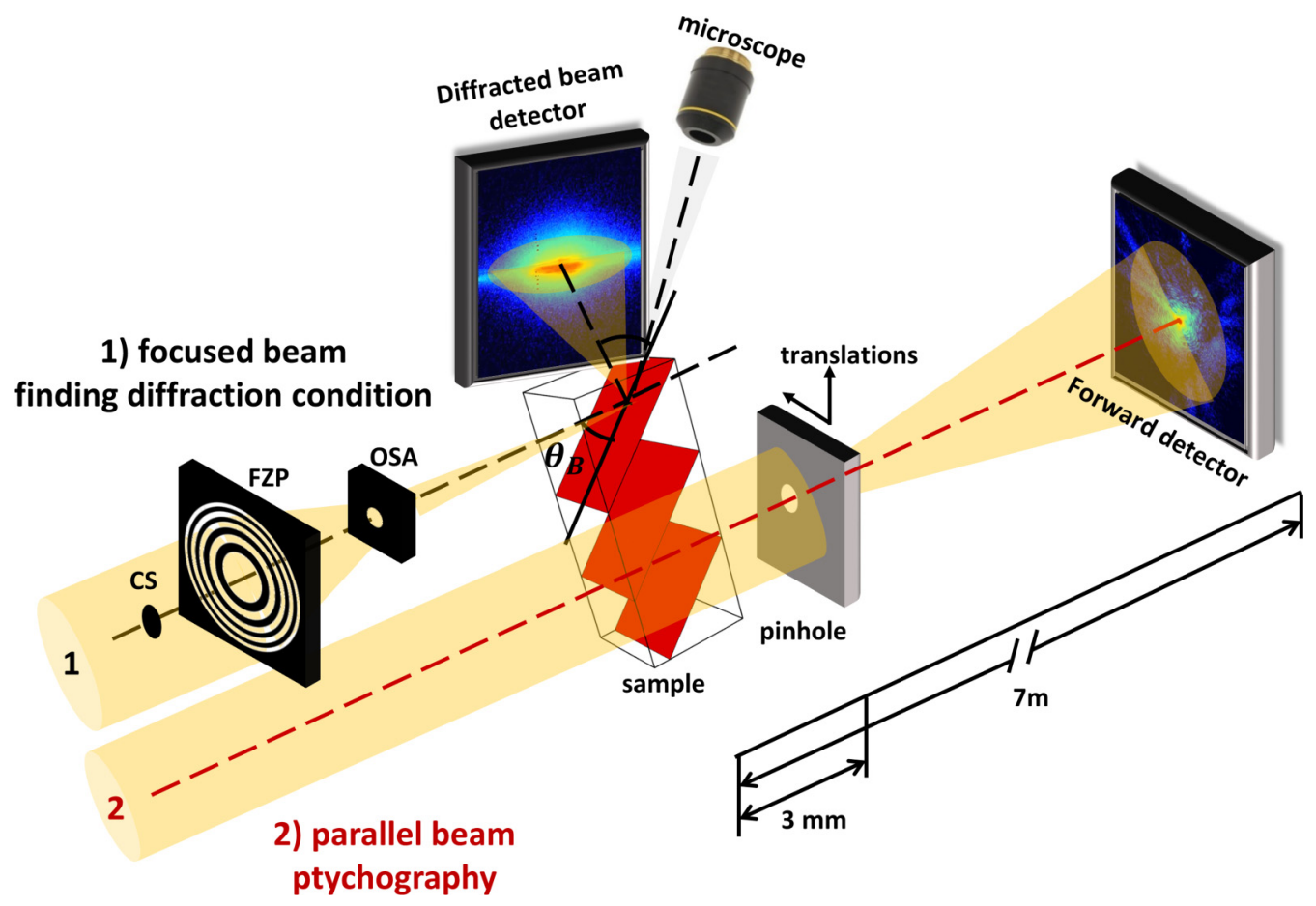

FIG. 1. Ptychographic topography acquisition scheme. Step (1) focused beam: sample alignment and diffraction peak detection from nonstrained crystal region using microscope, focusing optics, and 2D detector along the diffracted beam. Focusing optics include Fresnel zone plate (FZP), central stop (CS), and order-sorting aperture (OSA). Step (2) parallel beam: ptychographic topography with parallel beam. A pinhole is scanned after the sample and far-field patterns are recorded with the detector along the forward direction. Note: the two steps are performed consecutively.

requirements, but due to the divergence of the incoming beam the method is not working for crystals with lattice displacements within the scattering plane. In such cases, it is not always the same part of the convergent incoming beam that is diffracted, contradicting the hypothesis of a constant probe, as discussed in Ref. [17]. This reduces the robustness of the image reconstruction in Bragg ptychography.

Taking advantage simultaneously of the high strain sensitivity, large field of view, and simplicity of x-ray topography, as well as the high spatial resolution of x-ray ptychography, we propose an $\mathrm{x}$-ray imaging technique, ptychographic topography, for robust and flexible strain field imaging in extended crystalline samples with high spatial resolution. This method exploits the high strain sensitivity provided by a parallel, monochromatic incident beam in combination with the concept of tele-ptychography [17] to reconstruct the exit wave field a few millimeters downstream of the sample. Subsequent numerical backpropagation of the reconstructed wave field to the sample position provides the exit wave after parallel $x$ rays propagate through the crystal. This approach offers straightforward reconstructions, even in the case of complicated strain fields, with the resolution not limited by lenses.

In this paper, we describe the application of this technique to strain mapping of InSb micropillars that were uniaxially compressed up to the plastic deformation regime. The strain field is visualized in two different configurations of ptychographic topography, in forward and diffraction directions. The advantages and limitations of both configurations are explained and the effect of dynamical diffraction on the observed results is discussed.

\section{METHODS}

\section{A. The principle of ptychographic topography}

The concept of ptychographic topography consists of placing the crystal in diffraction condition for specific crystallographic planes and performing ptychographic scans in the forward (or diffraction) direction with a pinhole scanned several millimeters after the sample to reconstruct the exit wave field at the pinhole position. The resulting image is then numerically backpropagated to the crystal position and is sensitive to lattice imperfections.

As shown in Fig. 1, first the sample's region of interest is selected via an auxiliary visible light microscope. While setting up the experiment, we used a focused beam configuration to illuminate a region of the sample which has no crystalline defects, and to find and center the diffraction peak on the detector placed along the diffracted beam as shown in step (1) in Fig. 1.

To perform the ptychographic topography measurement, we switch to the parallel beam configuration [step (2) in Fig. 1]. A pinhole is placed a few millimeters after the sample and is scanned perpendicular to the beam, providing the sufficient overlap of the pinhole area at neighboring scan positions required for ptychographic reconstructions $[17,18]$. The diffraction patterns are recorded with a 2D detector downstream of the pinhole along the forward (or diffraction) direction in the far field and are used for the reconstruction of the wavefront at the pinhole position using ptychographic phase retrieval. Numerical backpropagation to the sample plane then results in an image of the sample sensitive to the lattice displacements caused by defects. 

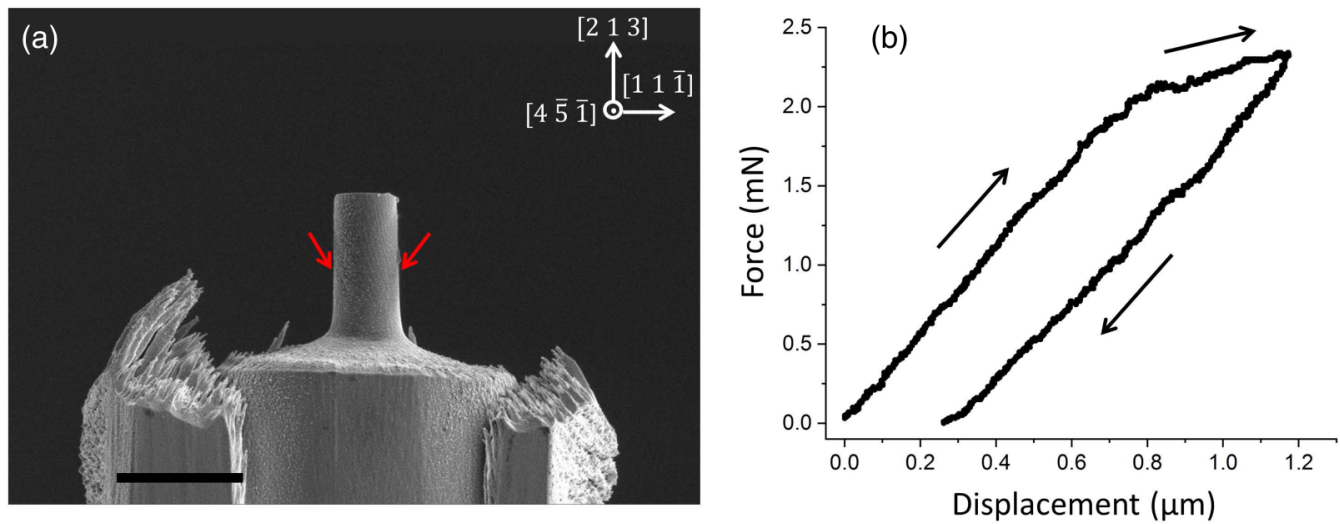

FIG. 2. Mechanical deformation of InSb micropillars. (a) SEM image of the pillar and its pedestal after uniaxial compression along the [2 13 3] crystallographic direction to reach the plastic deformation regime (applied force of $2.35 \mathrm{mN}$ ). Scale bar: $5 \mu \mathrm{m}$. (b) Force-displacement curve of the compression test that confirms that the sample was plastically deformed.

By rotating the sample and performing measurements at different angular steps close to a diffraction condition, quantitative information about the amount of strain and lattice rotations can be obtained. To recover all components of the three-dimensional displacement field one would need to measure at several nonequivalent diffraction peaks.

\section{B. InSb micropillars upon uniaxial compression}

Focused ion beam (FIB) milled InSb single-crystalline cylindrical micropillars were used to demonstrate the potential of the method. The samples were prepared by ion milling with $\mathrm{Ga}+$ focused ion beam (FIB) on an InSb single-crystalline wedge with known orientation in the form of a series of cylindrically shaped micropillars on cylindrical pedestals. The pillars dimensions are $2 \mu \mathrm{m}$ in diameter and $6 \mu \mathrm{m}$ in height. The pedestals dimensions are $10 \mu \mathrm{m}$ in diameter and $10 \mu \mathrm{m}$ in height. A scanning electron microscopy (SEM) image of such a pillar is shown in Fig. 2(a). Two equivalent pillars (S1 and S2) were investigated by ptychographic topography for reproducibility. The results from sample S1 are presented below while those from sample S2 are shown in the Supplemental Material [19].

The micropillars were uniaxially compressed with a dedicated microcompression device [20] along the pillar vertical axis that corresponds to the [l $\left.\begin{array}{lll}2 & 1 & 3\end{array}\right]$ direction, favoring only one dislocation slip system [21]. The pillars were compressed up to a force of $2.35 \mathrm{mN}$ (S1) and $2.5 \mathrm{mN}$ (S2), corresponding to the plastic regime, which led to an irreversible reduction of the pillars height of about $300 \mathrm{~nm}$ (S1) and $500 \mathrm{~nm}$ (S2) (i.e., about $5 \%$ and $8 \%$ of their initial height, respectively). This can be seen as a residual displacement after unloading at the force-displacement curve shown in Fig. 2(b). In addition, slip traces [pointed to with red arrows in Fig. 2(a)] are visible at the pillar surface.

\section{PTYCHOGRAPHIC TOPOGRAPHY IN FORWARD AND DIFFRACTION GEOMETRY}

We demonstrate the implementation of ptychographic topography in both the forward and diffraction direction at the cSAXS beamline at the Swiss Light Source (SLS). In both cases, we first used the focused beam configuration. We used a Fresnel zone plate of $200 \mu \mathrm{m}$ diameter and $90 \mathrm{~nm}$ outermost zone width upstream of the sample with a central stop of $60 \mu \mathrm{m}$ diameter and an order-sorting aperture of $30 \mu \mathrm{m}$ diameter. Such optical layout allowed achieving an illumination spot size of $\sim 100 \mathrm{~nm}$ in diameter. InSb samples were then brought to the $\left(\begin{array}{lll}2 & 0 & 2\end{array}\right)$ diffraction condition (similar to the geometry in Ref. [22]) at $6.2 \mathrm{keV}$, corresponding to a Bragg angle $\theta_{B}=25.88^{\circ}$ and an orientation angle of the sample $\omega \sim 7^{\circ}$, as shown in Fig. 3(a) and Fig. 3(d).

The region well below the pillar, i.e., the pedestal, was chosen for the diffraction peak alignment as a region without crystalline defects. The rocking curves were recorded with an Eiger $500 \mathrm{~K}$ detector [23] placed along the diffraction direction and a Pilatus $2 \mathrm{M}$ detector [24] along the forward direction. The rocking curves obtained at the pedestal position are shown in Fig. 3(c) and Fig. 3(f).

For forward ptychographic topography, the setup was then switched to the parallel-beam configuration, in which the beam is partially coherent with a transversal coherence of about $300 \mu \mathrm{m}$ in the vertical direction and $30 \mu \mathrm{m}$ in the horizontal direction. For this purpose we removed the focusing optics and placed a pinhole of $\sim 3.5 \mu \mathrm{m}$ diameter on a $2 \mathrm{D}$ piezo translation stage $4.5 \mathrm{~mm}$ downstream from the sample in the forward direction. Samples were rocked around the (2 0 2) diffraction peak in a range of $\pm 0.7^{\circ}$ with a step size of $0.01^{\circ}$. At each rocking angle, the pinhole was scanned after the sample perpendicular to the incoming beam with a step size of $0.5 \mu \mathrm{m}$ and $0.2 \mathrm{~s}$ exposure time covering a field of view of $12 \times 12 \mu \mathrm{m}^{2}$. The scanning positions followed a Fermat spiral pattern [25]. The diffraction patterns were recorded with a Pilatus $2 \mathrm{M}$ detector, placed at $7.338 \mathrm{~m}$ from the sample in the forward direction. The schematic of the described experiment is shown in Fig. 3(a).

The second experiment was performed in the diffraction geometry, using the parallel-beam configuration, a $2 \mu \mathrm{m}$ pinhole carried by the 2D piezo motors was placed at $2.2 \mathrm{~mm}$ downstream from the sample in the diffracted beam direction and used for scanning, see schematic in Fig. 3(d). The smaller, $2 \mu \mathrm{m}$ pinhole was used for appropriate sampling for ptychography at $2.171 \mathrm{~m}$. The data was acquired with the Eiger $500 \mathrm{~K}$ detector, placed at $2.171 \mathrm{~m}$ along the diffracted beam direction. Samples were rocked around the $\left(\begin{array}{lll}2 & 0 & 2\end{array}\right)$ 

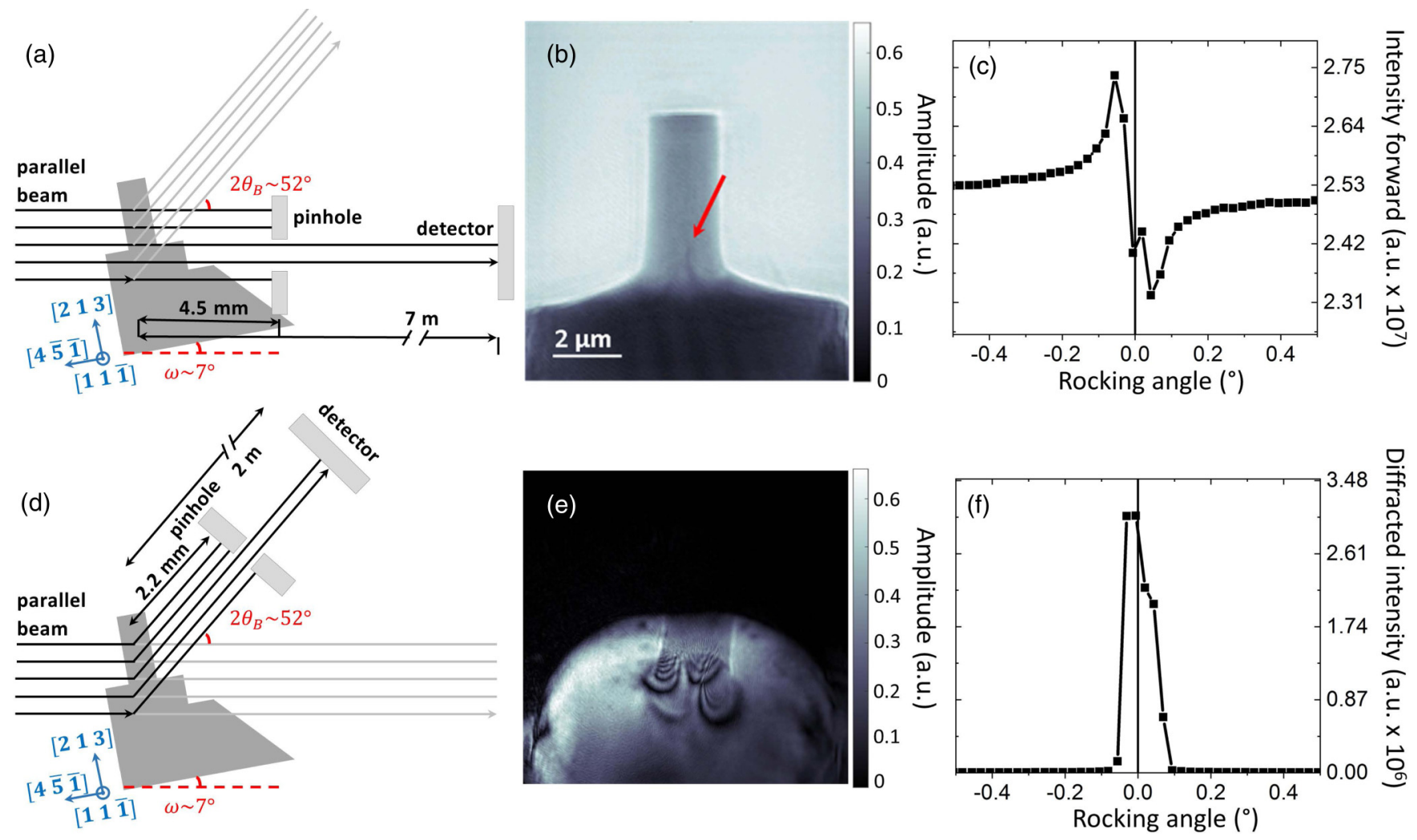

FIG. 3. Ptychographic topography of InSb micropillars in forward and diffraction directions. Schemes of ptychographic topography in the (a) forward and (d) diffraction direction with sample orientation (sample not to scale). (b) Amplitude of the image reconstruction obtained in the forward direction at the rocking angle of $0.03^{\circ}$. Red arrow shows strained region on the image. (c) Forward direction rocking curve around the (2 $\left.\begin{array}{ll}0 & 2\end{array}\right)$ diffraction peak measured with focused beam at the pedestal position. (e) Amplitude of the image reconstruction in the diffraction direction at the rocking angle of $0.002^{\circ}$. (f) Rocking curve in the diffraction direction around the (2 02 ) diffraction peak measured with focused beam at the pedestal position.

diffraction peak in a range of $\pm 0.02^{\circ}$ with an angular step of $0.002^{\circ}$. Ptychography scans were performed with a step size of $0.5 \mu \mathrm{m}$ and $0.2 \mathrm{~s}$ exposure time following a Fermat spiral pattern [25].

Ptychographic reconstructions were performed using 300 iterations of the difference map algorithm [26] followed by 1000 iterations of a maximum likelihood refinement [27] using the PtychoShelves package [28]. The resulting ptychographic reconstructions with a pixel size of $21 \mathrm{~nm}$ (in forward geometry) and $30 \mathrm{~nm}$ (in diffraction geometry) were backpropagated to the sample position and followed by postprocessing (phase offset and a linear phase wrap removal, vertical and horizontal spatial alignment) [29]. We estimated the resulting spatial resolution using Fourier ring correlation [30] (see Fig. S2 in the Supplemental Material [19]).

\section{STRAIN AND DYNAMICAL DIFFRACTION EFFECTS IN INSB}

As mentioned above, the 2D ptychographic projections were taken at different angular positions in the vicinity of the InSb (2 0 2 2) diffraction peak. This reflection is in Laue diffraction geometry, in which the diffracted wave exits the crystal at the opposite surface to the incoming beam [31]. The rocking curve was acquired with a focused beam in the forward direction at the pedestal position where we expect only negligible strain [Fig. 3(c)]. The curve shows increased intensity at angles just below the diffraction condition and exhibits an intensity drop at the diffraction angle. Such effect shows that dynamical scattering effects take place in this region of the crystal. This is expected as the pedestal is $10 \mu \mathrm{m}$ thick and the extinction length of InSb at $6.2 \mathrm{keV}$ and $\left(\begin{array}{ll}2 & 0\end{array}\right)$ diffraction reflection is $4.98 \mu \mathrm{m}$, as estimated from Ref. [32]. Such an effect is well known as a dynamical diffraction effect $[33,34]$, and it highlights the good crystallinity at the pedestal far away from the strained micropillar.

Each projection of the ptychographic topography experiment in the forward direction was reconstructed using single-probe mode [35] in order to reconstruct the pinhole, see Fig. S1(a). The fact of reconstructing a single probe mode is a sign that the reconstruction works well and that experimental parameters such as coherence and mechanical vibrations are well behaved to perform ptychography. The amplitude [Fig. 3(b)] of the ptychographic reconstruction clearly shows the pillar and pedestal shapes. At the bottom of the pillar and at the pillar/pedestal interface, a dark feature is visible, indicated by the red arrow in Fig. 3(b). This feature is only visible in the vicinity of the diffraction peak angle (as shown in Fig. 4 for rocking angles $0.01-0.04^{\circ}$ ) and is not observed far away from the diffraction peak (as shown in Fig. 4 for a rocking angle of $-3.5^{\circ}$ ). All projections can also be accessed in the Media S1. This feature corresponds to crystallinity 

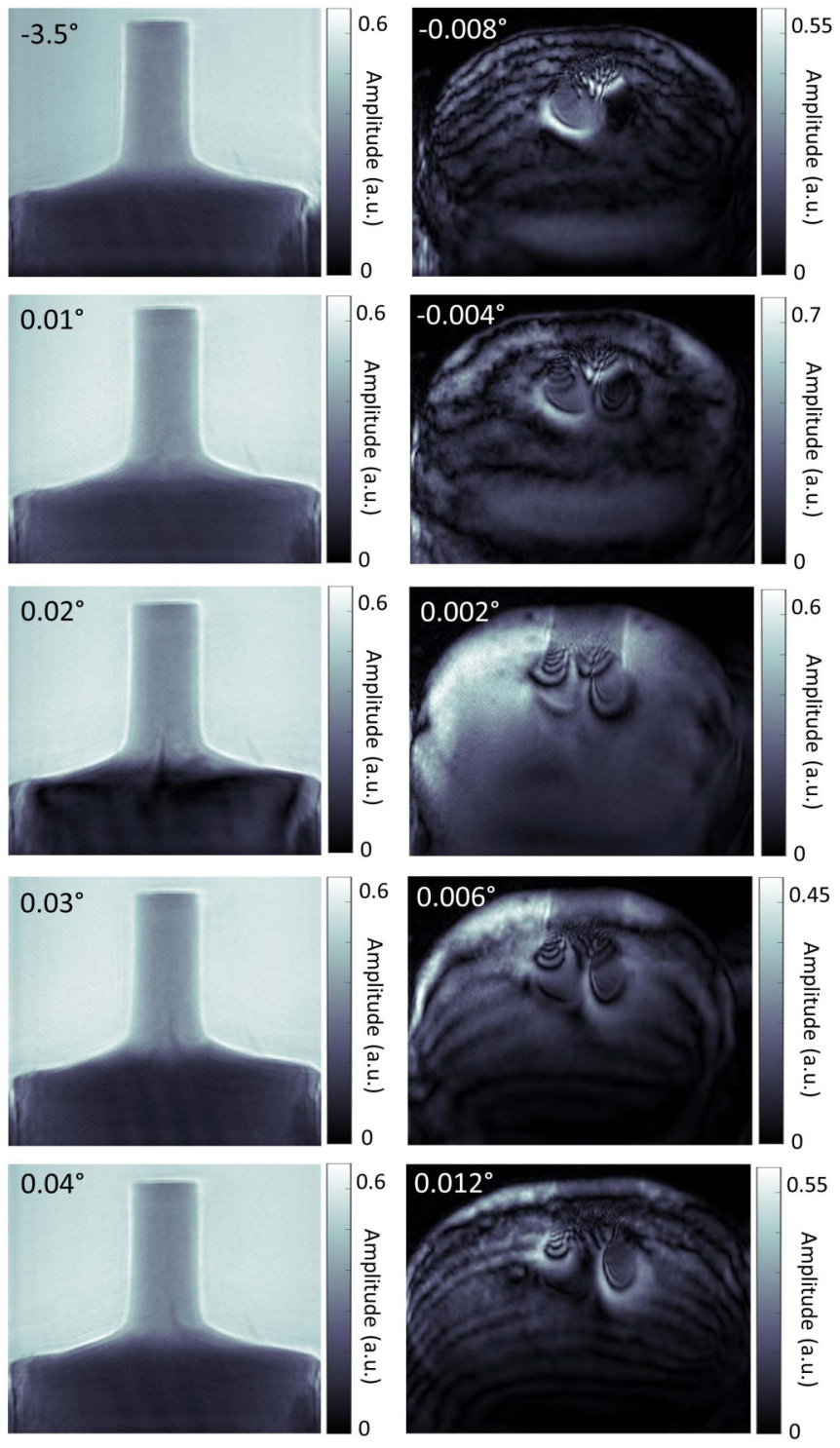

FIG. 4. Evolution of ptychographic topography of InSb micropillars in forward and diffraction geometry with the rocking angle around the (lll $\left.\begin{array}{ll}2 & 2\end{array}\right)$ reflection. The rocking angle is shown in the top left corner of individual images. Left column: amplitudes of the image reconstruction obtained in the forward direction. The image at the rocking angle of $-3.5^{\circ}$ is acquired far away from the diffraction condition and shows no strain features. Other images in the vicinity of the diffraction condition show the strain feature. Right column: amplitudes of the image reconstruction obtained in the diffraction direction, showing circular features and fringes of varying contrast.

imperfection or strain field present in the crystal that makes this part of the crystal diffract at a slightly different angle. The resolution of the reconstructed amplitude image was estimated to be $29 \mathrm{~nm}$.

It is also important to note that in ptychographic topography the amplitude provides higher contrast of crystalline imperfections compared to the phase reconstruction, as can be seen in Fig. S3 in the Supplemental Material [19]. This can be understood as a result of the diffraction-based contrast, similar to the one in x-ray topography. Nevertheless, we note that the phase information is needed for the backpropagation of the exit wave field from the pinhole to the sample position.

In the case of ptychographic topography in the diffraction direction, the reconstruction improved taking into account a second incoherent mode [35], with an intensity distribution for the first and second modes of $77 \%$ and $23 \%$, respectively [see in Fig. S1(b) and Fig. S1(c)]. This is similar to what is observed with Bragg ptychography as the reconstructions are more difficult compared to conventional ptychography and sometimes require several incoherent modes. As the signal in the diffraction direction is often several orders of magnitude smaller than in the direct transmitted beam, there is less data available while the system might also have higher stability requirements, which affects the robustness of the reconstruction.

The resulting reconstruction along the diffraction direction [Fig. 3(e)] contains no information at the top half of the images [black region in Fig. 3(e)]. This is partially due to the diffraction angle $2 \theta_{B} \sim 52^{\circ}$ at which those projections were recorded. At this angle, we see a projection of the whole pillar/pedestal assembly along the diffracted beam direction. In order to illustrate this effect, we calculated the projected thickness of the sample, including pillar and pedestal, along both the forward and diffraction direction in our measurement geometry (see Fig. S4 in the Supplemental Material [19]). The projected thickness of the sample, shown in Fig. S4(d), has a similar shape as observed in the image reconstructions along the diffraction direction and shows that the information of the whole pillar as well as the pedestal is encoded in the bottom half of the reconstructed image. Apart from this, no signal is expected to come from the top of the pillar as this region may have a very high density of defects and is out of diffraction condition, as will be discussed later.

A very interesting feature can be seen in the ptychographic topography reconstructions obtained in diffraction geometry [see Fig. 3(e)]: two sets of circular fringes of varying contrast. These features strongly change with the rocking angle and more interferences appear as we go further away from the diffraction angle, as shown in Fig. 4, right column. The projections at all rocking angles obtained by ptychographic topography in the diffraction direction can be seen in Media S2.

The interpretation of these features is complicated as it can involve the simultaneous presence of different effects. First of all, the thickness variation of single crystals can cause so-called Pendellösung fringes in the presence of dynamical scattering, i.e., if the crystal thickness is larger than the extinction length. This effect is widely observed in x-ray topography caused by thickness variations [36]. We believe that the thickness-based Pendellösung effect does not cause the observed fringes at the diffraction direction [Fig. 3(e)] as the projected thickness variation in the diffraction direction does not show similar patterns or significant sudden thickness change at the base of the pillar. However, thickness-based Pendellösung effect can very well explain the appearance and the shape of the additional fringes in the region of the pedestal as we go further away from the diffraction peak, as shown in Fig. 4, right column.

To confirm our hypothesis, we qualitatively simulated the absorption signal from the projected thicknesses using pencilbeam symmetrical Laue dynamical theory based on Eq. (16) 
(a)

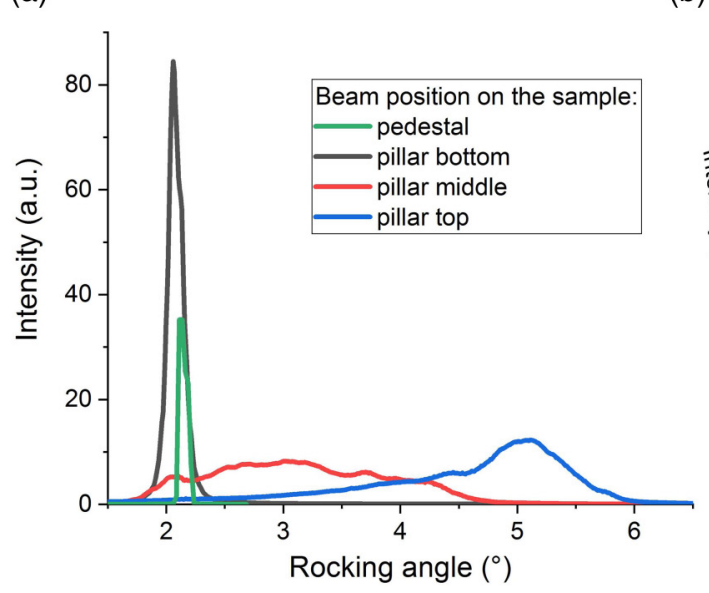

(b)

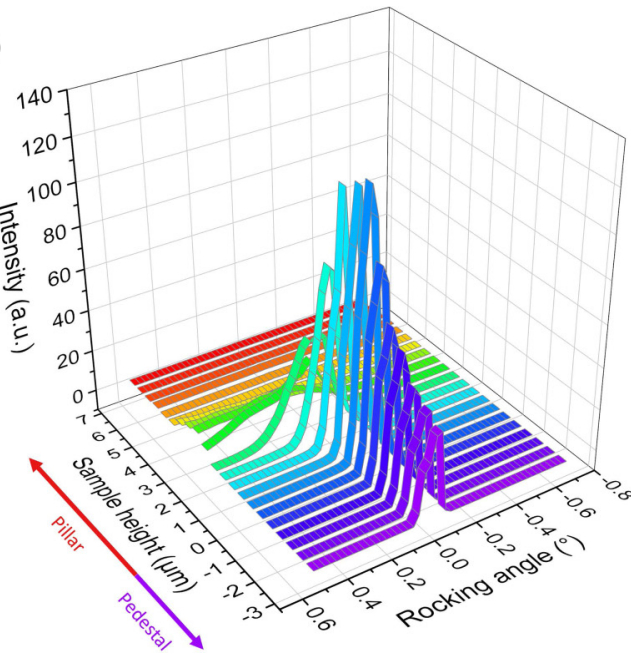

FIG. 5. Intensity of the $\left(\begin{array}{lll}2 & 0 & 2\end{array}\right)$ diffraction peak integrated over all pixels of the detector as a function of rocking angle, acquired with focused beam: (a) comparison of integrated intensity on the diffraction peak at four characteristic positions (at the pedestal, bottom, middle, and top of the pillar), (b) the (2 02 2) diffraction peak evolution along the pillar and pedestal height. Position of 0 height correspond to the pillar/pedestal interface.

in Ref. [37]. At some rocking angles, we were able to observe similarities between these fringes that appear further away from the diffraction condition with the simulated ones (reported in the Supplemental Material, Fig. S5 [19]). More detailed simulation, taking into account the nonsymmetric Laue dynamical theory as well as nonpencil beam is required to further understand this phenomenon but is beyond the scope of this paper.

A second possible effect, which could not be properly taken into account in our simulations, is the possibility of additional destructive and constructive interferences that emerge due to the beam path differences caused by our large nonfocused incident beam, so called Borrmann-Lehmann interference patterns [38,39]. This effect, which is also caused by dynamical scattering, could very well contribute to the observed fringes.

Finally, strain can also cause interference fringes. This effect is extensively studied with $\mathrm{x}$-ray topography using different single crystalline specimens with different strain states [40-43]. We believe that the fringes we observe with ptychographic topography in the diffraction direction are mainly caused by the strain present in the crystal in combination with the interference effects caused by the shape, thickness, and orientation of our sample. In addition, any interference fringes caused by sample size and shape are extremely sensitive to strain. The fringes symmetry, shape, and number will change in the presence of characteristic strain or lattice imperfections [39]. This makes ptychographic topography in the diffraction direction extremely sensitive to small crystalline imperfections and strain fields.

The stress-displacement curve in Fig. 2(b) shows that there has been a 5\% irreversible deformation in the total height of the pillar, which is a sign of considerable plastic deformation in the pillar. This was confirmed by SEM images performed after the compression experiment, like that shown in Fig. 2(a), which reveal the presence of slip traces, a typical signature of plastic deformation in this system [21]. Furthermore, a large amount of strain most probably in the form of lattice rotations is produced in addition to the slip planes, as confirmed by the $\mathrm{x}$-ray scanning diffraction microscopy below.

\section{ADDITIONAL CHARACTERIZATION BY SCANNING X-RAY MICROSCOPY}

To confirm our observations, we performed an additional sample characterization. First, using the $100 \mathrm{~nm}$ focused beam we performed angular rocking scans around the (2 02$)$ diffraction condition at different positions along the pillar (pedestal, pillar bottom, middle, and top), recording the intensity with the 2D detector along the diffraction direction, see Fig. 5(a). The rocking curves at the pedestal and bottom of the pillar are narrow, as expected for a crystal. But the curves for the middle and the top of the pillar show not only that the sample is out of the diffraction condition but also that the crystal may have too high density of defects to be reliably studied by monochromatic diffraction contrast methods. The linear scan along the pillar height in Fig. 5(b) also shows that the diffraction peak is the narrowest at the pedestal and is of sufficient quality at the bottom of the pillar, while the curve becomes broader and significantly shifted as the beam impinges higher on the pillar. Such a diffraction on the top of the pillar is another reason why we do not see the top of the pillar in Fig. 3(e).

Secondly, with the focused beam of $100 \mathrm{~nm}$ diameter, scanning step size of $100 \mathrm{~nm}$ and $0.1 \mathrm{~s}$ acquisition time, we performed scanning $\mathrm{x}$-ray diffraction microscopy at a fixed angle of $-0.05^{\circ}$ close to the $\left(\begin{array}{lll}2 & 0 & 2\end{array}\right)$ diffraction condition. Our findings are summarized in Fig. 6. The scanning transmission x-ray microscopy map in Fig. 6(a) shows no contrast to strain and the pillar appears brighter than the pedestal due to absorption. The scanning diffraction $\mathrm{x}$-ray microscopy map in Fig. 6(b) reveals strained areas within the pillar and at the pillar/pedestal interface. We also built the dark field image by masking out only the peak from the perfect crystal (pedestal position), see Fig. 6(c). By looking at individual diffraction patterns at different positions of the pillar and pedestal in Figs. 6(c) and 6(e) we conclude that the diffraction peak 

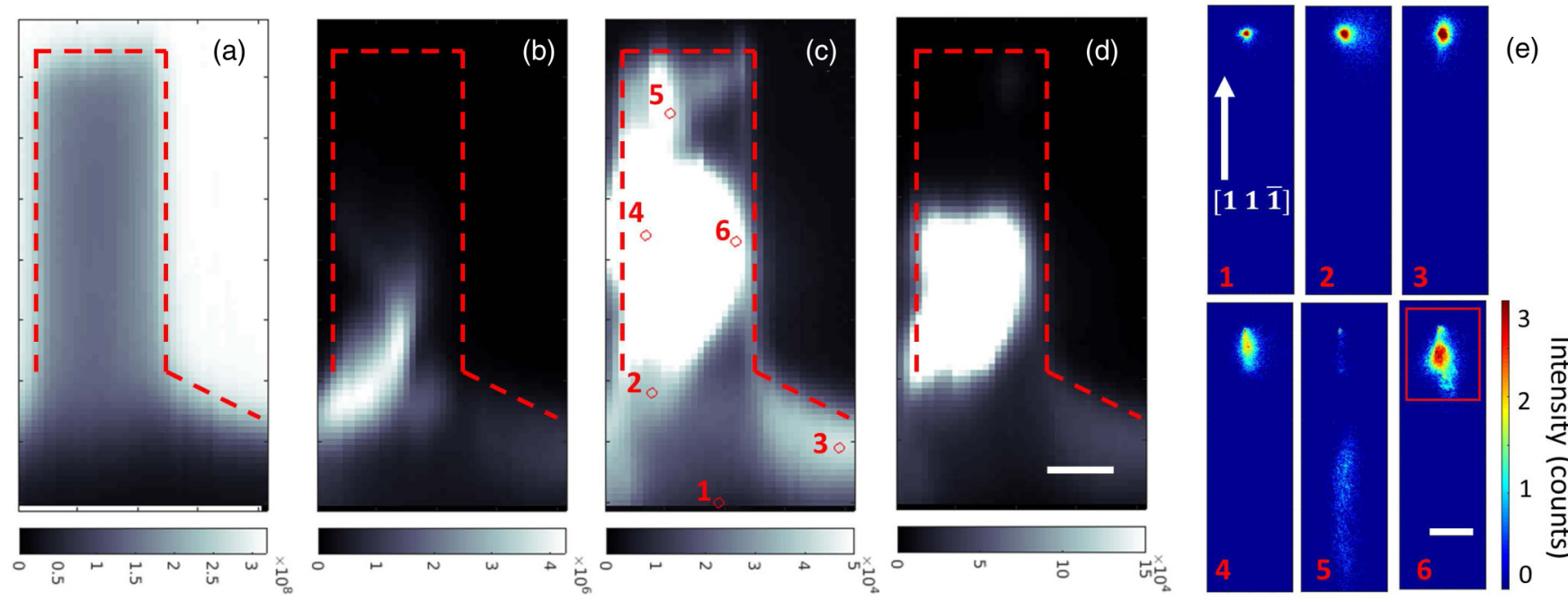

Intensity (counts)

FIG. 6. Scanning x-ray microscopy. (a) Intensity integrated over the central area around the beam of the forward detector. (b) Intensity integrated over the entire area of the detector at the diffraction direction. (c) Dark field image obtained by masking out only the peak from the perfect crystal (pedestal position 1) with characteristic diffraction patterns in (e). (d) Dark field image obtained by masking out the peak from the perfect crystal and using the ROI shown in (e) as a red rectangle. This ROI corresponds to the mild deformation, without taking into account very strong deformation in the form of peak splitting. Scale bar in (a)-(d): $1 \mu \mathrm{m}$. Scale bar in (e): $34.55 \mu \mathrm{m}^{-1}$.

begins broadening and changing shape at the top of the pedestal and bottom of the pillar (patterns 2 and 3), followed by vertical streaking in the middle of the pillar (patterns 4 and 6). But at the top half of the pillar (pattern 5) the diffraction peak streaks, splits, and moves out of the detector coverage (most of the peak intensity is no longer caught by the detector). Such a diffraction at the top of the pillar is a characteristic of a large deformation in this region and means that the sample has too high density of defects. Therefore, this method, as well as any other method that relies on monochromatic diffraction contrast, cannot provide the information to sufficiently describe strain in this region of the sample. Figure 6(d) shows another dark field image obtained by taking into account only the ROI that corresponds to a mild (i.e., measurable) deformation. From this image we can see that the bottom half of the pillar already has strong deformation, but higher resolution is needed to visualize individual details.

We summarize that scanning $\mathrm{x}$-ray diffraction confirms our observations with ptychographic topography that significant strain is present in the pillar and that the top half of the sample has too high density of defects. On the other hand, we would like to mention that ptychographic topography, while more experimentally demanding, has important benefits compared to scanning $\mathrm{x}$-ray diffraction microscopy: resolution not being limited to the lens and ability to simultaneously obtain phase information. In summary, our method provides a full description of the wavefront after the specimen, as phase information available in addition to the amplitude is present and is used to reconstruct the data and to propagate the object back to the sample position. This offers much more freedom of analyzing the data and correcting for potential artifacts. In addition, our method will benefit much more from the increase in brilliance at fourth generation synchrotrons and potentially be significantly faster than scanning microscopy measurements aiming for a similar resolution.

\section{DISCUSSION}

In the ptychographic topography demonstration described here, we observe deviations from perfect crystallinity due to strain and lattice rotations especially at the bottom part of the pillar, both along the forward and the diffraction directions. With a parallel incident beam, it is clear that a certain degree of crystallinity within the sample is needed in order to observe deviations from that crystallinity, and this is the case at the bottom part of the pillar. On the other hand, on the top part of the pillar the strain affects most of the bulk of the sample, in such a way that only a small sample volume diffracts at a given angle. Hence, for heavily strained crystals, the diffraction signal is too weak to be reconstructed in the diffraction geometry, and the diffraction-based contrast is too low in the forward geometry. Nevertheless, the advantage of having a parallel beam is that the technique can potentially have a very high sensitivity to strain in a crystalline specimen. As the contrast is the same as in x-ray topography, we expect that the strain sensitivity could be as good as $\Delta d / d=10^{-7}$, where $d$ is the lattice spacing of the crystal and $\Delta d$ is a change in $d$ over the initial length.

For illustration of reproducibility, the same ptychographic topography measurement was conducted along the $\left(\begin{array}{lll}2 & 0 & 2\end{array}\right)$ diffraction direction on a similar pillar, sample S2, that had also been previously mechanically compressed, see Fig. S6. The reconstructed amplitudes along the diffraction direction show many similarities in the type, contrast, and spacing of the fringes, although their exact arrangement is not identical and could be explained by different strain and/or lattice rotation distribution inside the pillar. Sample S2 was also investigated by ptychographic topography at the (2 20 ) Bragg condition along the forward direction (see Fig. S7), revealing at some angles a crystalline imperfection feature [Fig. S7(b)] similar to that observed in sample S1 at the (2 0 2) diffraction condition 
[Fig. 3(b)]. At a different angle, other interesting features were also observed [Fig. S7(c)]. However, the exact determination of the type of defects seen here will require further studies.

In this paper, we report a robust strain imaging technique, ptychographic topography, as well as its realization in both the forward and diffraction direction. This approach allows visualization of the exit wave after propagation of a beam through the crystal with high resolution (at least $29 \mathrm{~nm}$ as achieved in the present work) and both amplitude and phase contrast. In the forward direction, we obtain a superposition of the strained areas within the sample and the transmission image of the specimen, which is useful to study strain in a crystal in context with its surrounding material, regardless of its structural nature. In the diffraction direction, we observed fringes caused by dynamical scattering effects due to strain and geometrical features in the sample both in absorption and phase contrast. The use of a parallel beam offers opportunities for very high strain sensitivity when used on samples that present a sufficient degree of crystallinity. Signal-to-noise in ptychographic topography could be improved for such highly strained samples with the use of a coherently illuminated condenser designed to produce a flat illumination (i.e., providing a parallel beam) for $\mathrm{x}$-ray transmission microscopes [44]. With the currently available coherent flux in third generation synchrotron sources like the SLS, we estimate that coherent flux could be improved by about a factor of 10 already now by using this technology. With upcoming upgrades of synchrotron storage rings, an additional significant increase in the coherent flux will be possible. Most importantly, the simultaneous phase and amplitude reconstruction provided by our method opens a potential to retrieve quantitative strain field directly from the topography data.

\section{ACKNOWLEDGMENTS}

We acknowledge the Paul Scherrer Institute, Villigen, Switzerland for provision of synchrotron radiation beamtime at the cSAXS beamline of the SLS. This work was supported by the SNF Grant No. 200021L_169753, ANR-16-CE93-0006, by "Investissement d'Avenir" (LABEX INTERACTIFS, ANR-11-LABX-0017-01) and by Nouvelle Aquitaine Region/European Structural and Investment Funds (ERDF No P-2016-BAFE-94/95). M.V. was supported by the European Union's Horizon 2020 research and innovation program under the Marie Skłodowska-Curie Grant Agreement No. 701647. K.W. acknowledges the support by the SNF Grant No. 200021_166304. D.K. acknowledges funding from SNSF under Grant No. 200021_175905. Authors thank M. Guizar-Sicairos, P. O. Renault, T. Sadat, D. Le Bolloc'h, B. Kedjar, and F. Mignerot for fruitful discussions.
[1] M. J. Hÿtch and A. M. Minor, Observing and measuring strain in nanostructures and devices with transmission electron microscopy, MRS Bull. 39, 138 (2014).

[2] N. Hrauda, J. Zhang, E. Wintersberger, T. Etzelstorfer, B. Mandl, J. Stangl, D. Carbone, V. Holy, V. Jovanovic, C. Biasotto, L. Nanver, J. Moers, D. Grutzmacher, and G. Bauer, $\mathrm{X}$-ray nanodiffraction on a single sige quantum dot inside a functioning field-effect transistor, Nano Lett. 11, 2875 (2011).

[3] G. N. Ramachandran, X-Ray topographs of diamond, Proc. Indian Acad. Sci. Sect. A 19, 280 (1944).

[4] Y. Tsusaka, S. Takeda, H. Takano, K. Yokoyama, Y. Kagoshima, and J. Matsui, X-ray topography using the forward transmitted beam under multiple-beam diffraction conditions, Rev. Sci. Instrum. 87, 023701 (2016).

[5] G. E. Ice and J. W. L. Pang, Tutorial on x-ray microLaue diffraction, Mater. Charact. 60, 1191 (2009).

[6] R. Maaß, S. Van Petegem, D. Ma, J. Zimmermann, D. Grolimund, F. Roters, H. Van Swygenhoven, and D. Raabe, Smaller is stronger: The effect of strain hardening, Acta Mater. 57, 5996 (2009).

[7] H. Simons, A. King, W. Ludwig, C. Detlefs, W. Pantleon, S. Schmidt, F. Stöhr, I. Snigireva, A. Snigirev, and H. F. Poulsen, Dark-field X-ray microscopy for multiscale structural characterization, Nat. Commun. 6, 6098 (2015).

[8] T. Etzelstorfer, M. J. Süess, G. L. Schiefler, V. L. R. Jacques, D. Carbone, D. Chrastina, G. Isella, R. Spolenak, J. Stangl, H. Sigg, and A. Diaz, Scanning X-ray strain microscopy of inhomogeneously strained Ge micro-bridges, J. Synchrotron Radiat. 21, 111 (2014).

[9] G. A. Chahine, M.-I. Richard, R. A. Homs-Regojo, T. N. Tran-Caliste, D. Carbone, V. L. R. Jacques, R. Grifone, P.
Boesecke, J. Katzer, I. Costina, H. Djazouli, T. Schroeder, and T. U. Schülli, Imaging of strain and lattice orientation by quick scanning X-ray microscopy combined with three-dimensional reciprocal space mapping, J. Appl. Crystallogr. 47, 762 (2014).

[10] V. L. R. Jacques, S. Ravy, D. Le Bolloc'h, E. Pinsolle, M. Sauvage-Simkin, and F. Livet, Bulk Dislocation Core Dissociation Probed by Coherent X Rays in Silicon, Phys. Rev. Lett. 106, 065502 (2011).

[11] J. Miao, T. Ishikawa, I. K. Robinson, and M. M. Murnane, Beyond crystallography: Diffractive imaging using coherent $\mathrm{X}$-ray light sources, Science 348, 530 (2015).

[12] F. Pfeiffer, X-ray ptychography, Nat. Photonics 12, 9 (2018).

[13] M. A. Pfeifer, G. J. Williams, I. A. Vartanyants, R. Harder, and I. K. Robinson, Three-dimensional mapping of a deformation field inside a nanocrystal, Nature (London) 442, 63 (2006).

[14] J. M. Rodenburg, A. C. Hurst, A. G. Cullis, B. R. Dobson, F. Pfeiffer, O. Bunk, C. David, K. Jefimovs, and I. Johnson, HardX-Ray Lensless Imaging of Extended Objects, Phys. Rev. Lett. 98, 034801 (2007).

[15] P. Godard, G. Carbone, M. Allain, F. Mastropietro, G. Chen, L. Capello, A. Diaz, T. Metzger, J. Stangl, and V. Chamard, Three-dimensional high-resolution quantitative microscopy of extended crystals, Nat. Commun. 2, 568 (2011).

[16] S. O. Hruszkewycz, M. Allain, M. V. Holt, C. E. Murray, J. R. Holt, P. H. Fuoss, and V. Chamard, High-resolution three-dimensional structural microscopy by single-angle Bragg ptychography, Nat. Mater. 16, 244 (2017).

[17] E. H. R. Tsai, A. Diaz, A. Menzel, and M. Guizar-Sicairos, X-ray ptychography using a distant analyzer, Opt. Express 24, 6441 (2016). 
[18] M. Verezhak, S. Van Petegem, V. Jacques, P. Godard, K. Wakonig, L. Thilly, and A. Diaz, Visualization of crystallographic defects in InSb micropillars by ptychographic topography, Microsc. Microanal. 24, 18 (2018).

[19] See Supplemental Material at http://link.aps.org/supplemental/ 10.1103/PhysRevB.103.144107 for ptychographic reconstructions of the pinholes, resolution estimated using Fourier ring correlation, comparison of amplitude and phase of the image reconstruction in the forward direction, simulations of the sample thickness along the beam path, pendellosung fringes simulations, sample s2, and medias of ptychographic topography reconstructions of $\mathrm{InSb}$ micropillar in the forward and diffraction direction at different angular positions of the rocking curve.

[20] C. Kirchlechner, J. Keckes, J.-S. Micha, and G. Dehm, In situ microLaue: Instrumental setup for the deformation of micron sized samples, Adv. Eng. Mater. 13, 837 (2011).

[21] L. Thilly, R. Ghisleni, C. Swistak, and J. Michler, In situ deformation of micro-objects as a tool to uncover the micro-mechanisms of the brittle-to-ductile transition in semiconductors: the case of indium antimonide, Philos. Mag. 92, 3315 (2012).

[22] V. L. R. Jacques, D. Carbone, R. Ghisleni, and L. Thilly, Counting Dislocations in Microcrystals by Coherent X-Ray Diffraction, Phys. Rev. Lett. 111, 065503 (2013).

[23] I. Johnson, A. Bergamaschi, H. Billich, S. Cartier, R. Dinapoli, D. Greiffenberg, M. Guizar-Sicairos, B. Henrich, J. Jungmann, D. Mezza, A. Mozzanica, B. Schmitt, X. Shi, and G. Tinti, Eiger: a single-photon counting X-ray detector, J. Instrum. 9, C05032 (2014).

[24] B. Henrich, A. Bergamaschi, C. Broennimann, R. Dinapoli, E. Eikenberry, I. Johnson, M. Kobas, P. Kraft, A. Mozzanica, and B. Schmitt, Pilatus: A single photon counting pixel detector for X-ray applications, Nucl. Instrum. Methods Phys. Res., Sect. A 607, 247 (2009), Radiation Imaging Detectors 2008.

[25] X. Huang, H. Yan, R. Harder, Y. Hwu, I. K. Robinson, and Y. S. Chu, Optimization of overlap uniformness for ptychography, Opt. Express 22, 12634 (2014).

[26] P. Thibault, M. Dierolf, O. Bunk, A. Menzel, and F. Pfeiffer, Probe retrieval in ptychographic coherent diffractive imaging, Ultramicroscopy 109, 338 (2009).

[27] P. Thibault and M. Guizar-Sicairos, Maximum-likelihood refinement for coherent diffractive imaging, New J. Phys. 14, 063004 (2012).

[28] K. Wakonig, H.-C. Stadler, M. Odstrčil, E. H. R. Tsai, A. Diaz, M. Holler, I. Usov, J. Raabe, A. Menzel, and M. GuizarSicairos, PtychoShelves, a versatile high-level framework for high-performance analysis of ptychographic data, J. Appl. Crystallogr. 53, 574 (2020).

[29] M. Guizar-Sicairos, A. Diaz, M. Holler, M. S. Lucas, A. Menzel, R. A. Wepf, and O. Bunk, Phase tomography from $\mathrm{x}$-ray coherent diffractive imaging projections, Opt. Express 19, 21345 (2011).

[30] M. van Heel and M. Schatz, Fourier shell correlation threshold criteria, J. Struct. Biol. 151, 250 (2005).

[31] A. Rodriguez-Fernandez, V. Esposito, D. F. Sanchez, K. D. Finkelstein, P. Juranic, U. Staub, D. Grolimund, S. Reiche, and B. Pedrini, Spatial displacement of forward-diffracted X-ray beams by perfect crystals, Acta Crystallogr., Sect. A: Found. Adv. 74, 75 (2018).

[32] A. Authier, Dynamical Theory of X-Ray Diffraction (Oxford University Press, 2010), pp. 626-646.

[33] W. H. Zachariasen, Theory of X-ray Diffraction in Crystals (Dover Publications, New York, 1968), p. 255.

[34] B. W. Batterman and H. Cole, Dynamical Diffraction of X Rays by Perfect Crystals, Rev. Mod. Phys. 36, 681 (1964).

[35] P. Thibault and A. Menzel, Reconstructing state mixtures from diffraction measurements, Nature (London) 494, 68 (2013).

[36] N. Kato and A. R. Lang, A study of pendellösung fringes in X-ray diffraction, Acta Crystallogr. 12, 787 (1959).

[37] V. I. Punegov, S. I. Kolosov, and K. M. Pavlov, BraggLaue X-ray dynamical diffraction on perfect and deformed lateral crystalline structures, J. Appl. Crystallogr. 49, 1190 (2016).

[38] Z. Mai and H. Zhao, Absorption effect on Borrmann-Lehmann interference phenomena, Acta Crystallogr., Sect. A: Found. Crystallogr. 45, 602 (1989).

[39] A. R. Lang, G. Kowalski, and A. P. W. Makepeace, BorrmannLehmann interference patterns in perfect and in distorted crystals, Acta Crystallogr., Sect. A: Found. Crystallogr. 46, 215 (1990).

[40] U. Bonse, M. Hart, and G. H. Schwuttke, X-ray investigation of lattice deformations in silicon induced through high-energy ion implantation, Phys. Status Solidi B 33, 361 (1969).

[41] R. Negishi, T. Fukamachi, M. Yoshizawa, K. Hirano, and T. Kawamura, Observation of interference fringes due to lattice distortion by resonant scattering X-ray topography, Phys. Status Solidi A 204, 2694 (2007).

[42] F. N. Chukhovskii and P. V. Petrashen, X-ray topography of bent crystals, Acta Crystallogr., Sect. A: Found. Crystallogr. 44, 8 (1988).

[43] S. M. Novikov, I. M. Fonchuk, D. G. Fedortsov, and A. Y. Struk, Calculated images of dislocations in crystals on section topograms, Semicond. Phys., Quantum Electron. Optoelectron. 13, 268 (2010).

[44] K. Jefimovs, J. Vila-Comamala, M. Stampanoni, B. Kaulich, and C. David, Beam-shaping condenser lenses for full-field transmission X-ray microscopy, J. Synchrotron Radiat. 15, 106 (2008). 